\title{
Development Plan for the MOX Fuel Fabrication Process
}

Nuclear Technology

Research and Development

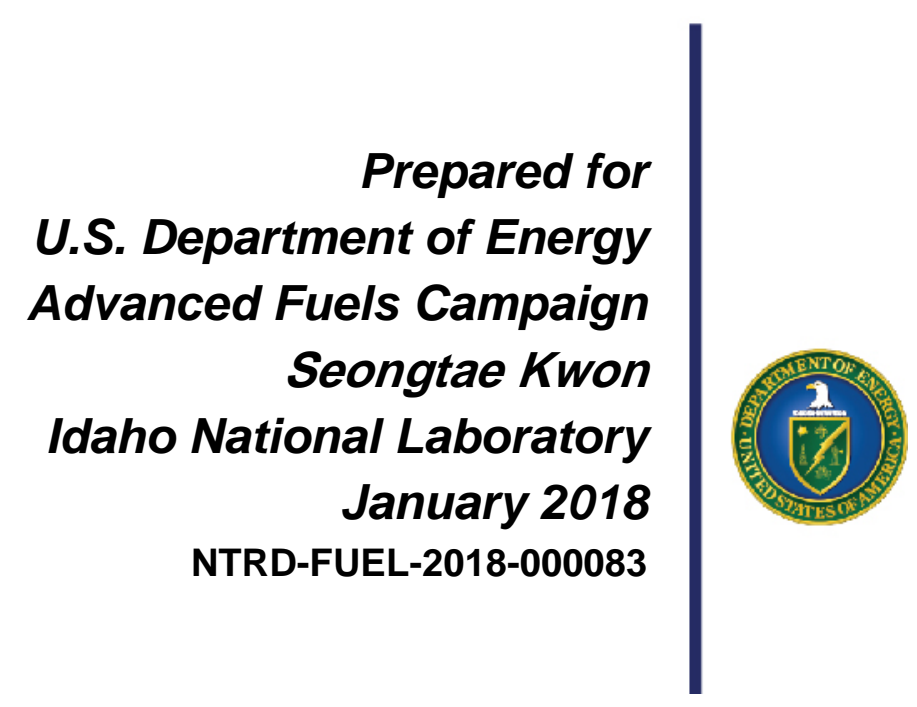




\section{DISCLAIMER}

This information was prepared as an account of work sponsored by an agency of the U.S. Government. Neither the U.S. Government nor any agency thereof, nor any of their employees, makes any warranty, expressed or implied, or assumes any legal liability or responsibility for the accuracy, completeness, or usefulness, of any information, apparatus, product, or process disclosed, or represents that its use would not infringe privately owned rights. References herein to any specific commercial product, process, or service by trade name, trade mark, manufacturer, or otherwise, does not necessarily constitute or imply its endorsement, recommendation, or favoring by the U.S. Government or any agency thereof. The views and opinions of authors expressed herein do not necessarily state or reflect those of the U.S. Government or any agency thereof. 


\section{SUMMARY}

The Advanced Fuel Campaign (AFC) in Fuel Cycle Research and Development (FCRD) has tasks of near term Accident Tolerant Fuel (ATF) for light water reactor (LWR) and long term advanced reactor fuel research and development. Mixed Oxide (MOX) is one of the options for the advanced reactor fuel, and the development plan for the fabrication and characterization of MOX is described. The efforts will include the confirmation of the MOX fabrication capability and development of processing parameters for the stable fabrication of MOX fuel to support future MOX work if the need arises from the program. Establishing a robust fabrication process of desired stoichiometry utilizing the fabrication equipment recently added to the Fuel Manufacturing Facility (FMF) glove box sintering furnace would be the main goal of the development. 
INTENTIONALLY BLANK 


\section{CONTENTS}

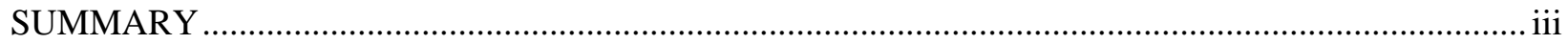

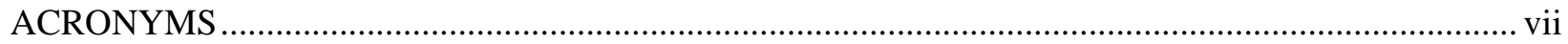

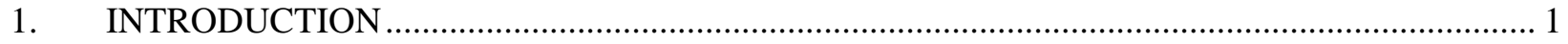

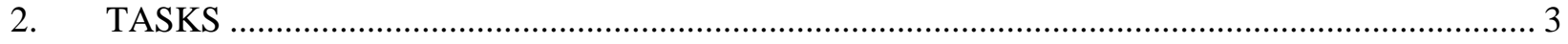

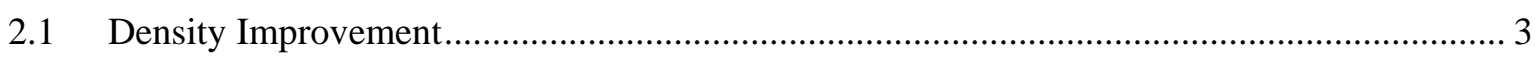

2.2 Sintering Atmosphere Reproducibility Confirmation ........................................................... 3

2.3 Oxygen Partial Pressure and Stoichiometry Relationship Map Development........................ 4

2.4 High Temperature Stable Microstructure Development .......................................................... 4

2.5 Stoichiometry Characterization Method Development........................................................ 5

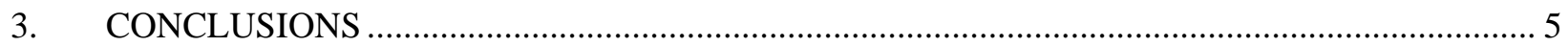

\section{FIGURES}

Figure 1. Processing record of the practice sintering run (a) temperature and set point and

(b) monitored oxygen partial pressure as a function of time. ................................................. 1

Figure 2. Pellets sintered during ATP (a) surrogate and (b) MOX...................................................... 2

Figure 3. Particle size analysis result of depleted $\mathrm{UO}_{2}$ powder being used.............................................. 3

\section{TABLES}

Table 1. Gas mixture and oxygen partial pressure during the practice sintering run................................. 2

Table 2. Gas mixture ratio and for $-350 \mathrm{~kJ} / \mathrm{mol}$ oxygen partial pressure for $\left(\mathrm{U}_{0.8} \mathrm{Pu}_{0.2}\right) \mathrm{O}_{2}$ composition at various temperatures. 
INTENTIONALLY BLANK 


\section{ACRONYMS}

AFC Advanced Fuel Campaign

ATF Accident Tolerant Fuel

ATP Acceptance Test Procedure

FCRD Fuel Cycle Research and Development

FMF Fuel Manufacturing Facility

INL Idaho National Laboratory

LWR light water reactor

MOX mixed oxide

TGA thermogravimetric analysis 
INTENTIONALLY BLANK 


\section{INTRODUCTION}

Idaho National Laboratory (INL) acquired sintering capability of MOX fuel in a glove box at the Fuel Manufacturing Facility (FMF) at the end of September 2017. The activities included installation of oxygen monitors for inlet and outlet of sintering furnace, installation of digital mass flow controller power supply, installation of wet argon line, and completion of the software to control the system. The capability of controlling oxygen partial pressure during the sintering of mixed oxide (MOX) fuel has been established by mixing $\mathrm{H}_{2} \mathrm{O} / \mathrm{H}_{2}$ ratio. Figure 1 illustrates the temperature and oxygen partial pressures measured from the inlet and out let of the sintering furnace during the practice run after new components and software are installed. There is a difference between the oxygen partial pressure inlet and outlet; less oxygen was detected from the outlet. It is believed that the samples along with the metal in the furnace functioned as an oxygen getter. The bumps at low temperature and fluctuation of the oxygen partial pressure will be further explored with repeated runs. Table 1 shows the processing parameter for the oxygen partial pressure control and compares the measured oxygen partial pressure and calculated value at $\sim 650^{\circ} \mathrm{C}$.

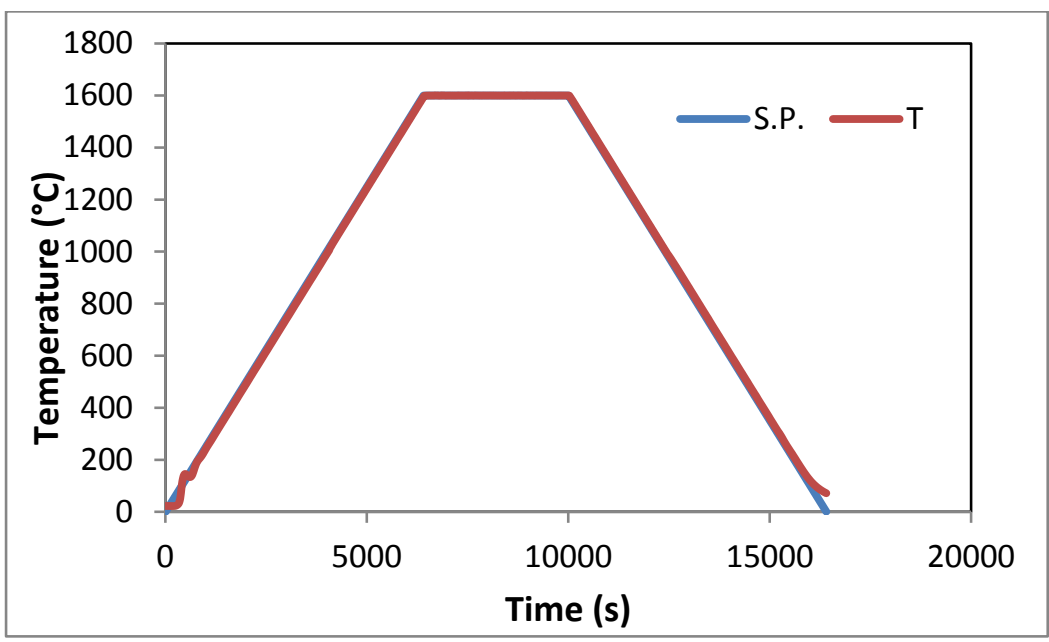

(a)

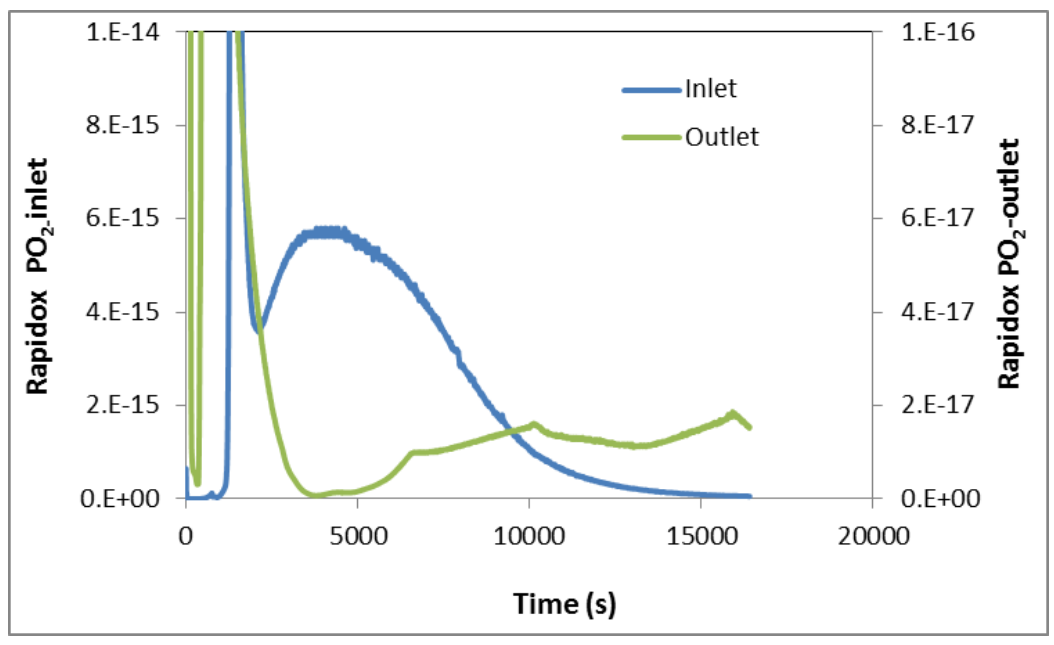

(b)

Figure 1. Processing record of the practice sintering run (a) temperature and set point and (b) monitored oxygen partial pressure as a function of time. 
Table 1. Gas mixture and oxygen partial pressure during the practice sintering run.

\begin{tabular}{|c|c|c|c|c|c|c|}
\hline & \multicolumn{3}{|c|}{ Gas flow (sccm) } & \multicolumn{3}{c|}{$\mathrm{PO}_{2}$} \\
\hline $\mathrm{T}\left({ }^{\circ} \mathrm{C}\right)$ & Dry Ar & $\mathrm{H}_{2}$ & wet $\mathrm{Ar}$ & Calculated & inlet & outlet \\
\hline 650.7 & 146.8 & 0.4 & 49.7 & $2.08 \mathrm{E}-27$ & $4.59 \mathrm{E}-21$ & $1.54 \mathrm{E}-23$ \\
\hline
\end{tabular}

A substoichiometric MOX fuel is essential for high burnup applications. Achieving a stable low oxygen partial pressure during sintering is a key factor in fabricating MOX fuels with substoichiometry. Through the Acceptance Test Procedure (ATP), it was confirmed that the furnace in FMF has the capability of proper temperatures and heating rate for the densification of MOX pellets. The capability of maintaining desired oxygen partial was also verified through a couple of sintering runs with surrogate, depleted $\mathrm{UO}_{2}$ and MOX pellets. Figure 2 shows the surrogate and MOX pellets sintered during the ATP runs at $1600^{\circ} \mathrm{C}$ for $1 \mathrm{hr}$.

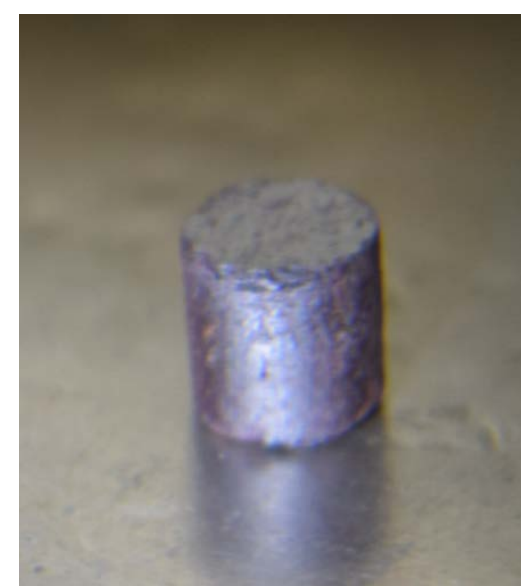

(a)

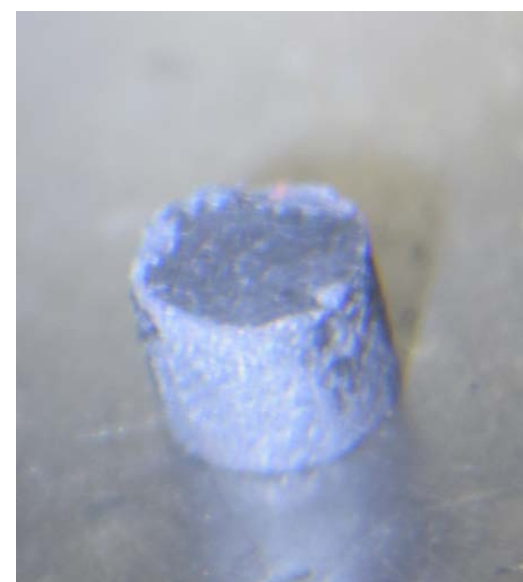

(b)

Figure 2. Pellets sintered during ATP (a) surrogate and (b) MOX.

The initial experiment resulted in low green density and, consequently, low sintered density at $~ 74 \%$ of the theoretical density. A systematical experiment is required to develop procedures for the fabrication of MOX fuels with desired physical properties such as density, stoichiometry, and microstructure.

The stoichiometry of the sample is to be determined using either thermogravimetric analysis (TGA) or a tube furnace methodology. The capability of controlling oxygen partial pressure in the same way as is used in the sintering furnace is required for the TGA analysis to determine the stoichiometry of sintered MOX pellets. Two different approaches will be attempted to develop the stoichiometry characterization. One approach will be adding oxygen partial pressure controlling and monitoring capability to the TGA currently installed in the Fresh Fuels Glovebox, and the second approach is determine the feasibility of high precision weight measurements in FMF glove box which currently has a tube furnace capable of atmosphere control and oxygen sensing.

High burnup is one of the main targets of the MOX fuel for a fast reactor. Substoichiometry to prevent corrosion of cladding from oxidation and relatively low pellet density (85 90\% of theoretical density) to accommodate fission product are two key properties needed to allow high burn-up capabilities.

Additionally, dimensional stability of the fuel during irradiation is necessary to maintain adequate thermal conductivity. It should be noted, because low pellet density and fuel dimension must be retained during the application of high temperatures (in excess of $2000^{\circ} \mathrm{C}$ ) that the required low fuel density cannot be 
simply achieved by insufficient sintering at low temperatures. Instead, a stable microstructure retaining low density at application temperatures is required.

\section{TASKS}

\subsection{Density Improvement}

The low sintered densities witnessed from the initial trials resulted from low green densities. It is believed that the pressing mold used for the initial trials, made of high speed steel, was not adequate in terms of hardness and dimensional tolerance.

Pressing punches and mold easily deteriorated as pressing went on and allowed powder to be squeezed between the mold and punch, which made cleaning the mold between the pressings difficult and caused low green densities ( $45 \%$ ) in spite of high pressing pressure, up to $3100 \mathrm{~kg} / \mathrm{cm}^{2}$.

A pressing mold with tighter tolerance between the die and punches made of hard material such as tungsten carbide (WC) is essential when it is considered that the powder preparation process is limited to dry-base process in the glove box. A new mold made of tungsten carbide is being procured and to be used in the pellet pressing. Surrogate samples will be pressed using depleted $\mathrm{UO}_{2}$ powder to compare the effect of the new mold with the previous pressing results. If the depleted $\mathrm{UO}_{2}$ pellets show substantial improvement then basic MOX composition, $\left(\mathrm{U}_{0.8} \mathrm{Pu}_{0.2}\right) \mathrm{O}_{2}$ will be further explored with the new mold. Green relative density higher than $50 \%$ will be the first stage target of this task. Other processing modification such as use of a new binder system will be determined using a progressive approach.

If the higher quality die and new binder system do not substantially improve the density wet mixing could be an option for the next stage. Use of organic solvent could be a challenging issue in the glove box, but can be discussed to provide homogeneous mixing of the binder along with communion of detrimental aggregates in the raw powder. The particle size analysis of the depleted $\mathrm{UO}_{2}$ powder is shown in Figure 3. The powder turned out to have a slight bimodal distribution with 4.9 um mean, 0.56 um mode and 0.74 um median particle size. In case the removal of aggregate is necessary from the raw powder, wet mixing process such as ball milling using high purity stabilized zirconia grinding media in an organic solvent will be considered.

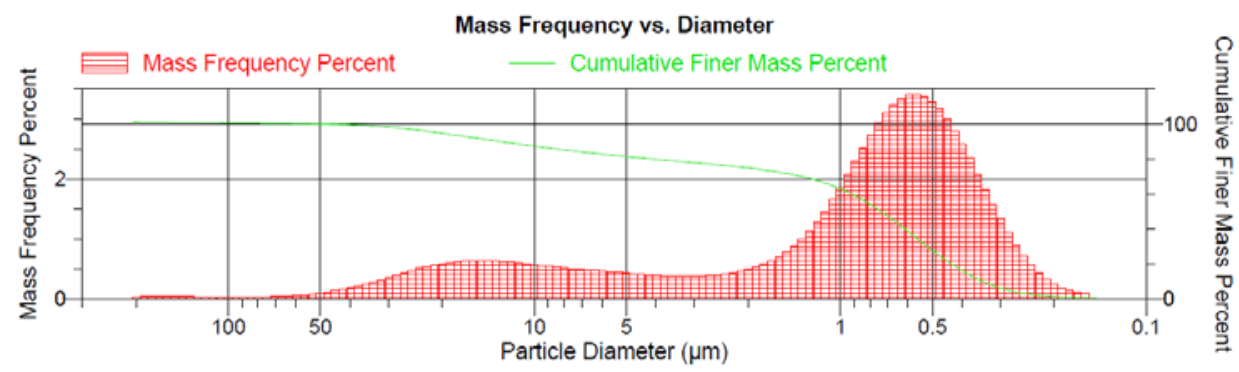

Figure 3. Particle size analysis result of depleted $\mathrm{UO}_{2}$ powder being used.

\subsection{Sintering Atmosphere Reproducibility Confirmation}

From the initial sintering runs with mixed wet $\mathrm{Ar}$ and $\mathrm{H}_{2}$, and in pure dry Ar, it was seen that an oxygen partial pressure of $10^{-25} \sim 10^{-23}$ was achieved at $650^{\circ} \mathrm{C}$. The reproducibility of the oxygen partial pressure will be further investigated in the repeated sintering runs. Key sintering parameters such as flow amount of gases, temperature, monitored oxygen partial pressure, and humidity will be all recorded in the data file for each sintering run. The checking points will include;

- change of oxygen partial pressure with temperature and time during the run

- stability of inlet/outlet oxygen partial pressure within and between runs 
- reproducibility of oxygen partial pressure run to run

- comparison of monitored oxygen partial pressure with calculated values from $\mathrm{H}_{2} \mathrm{O} / \mathrm{H}_{2}$ ratio

- confirmation of the moisture content in nitrogen gas and its utilization for higher oxygen partial pressure ranges beyond wet Ar content.

Feasibility of achieving stable sintering atmosphere will be confirmed by repeated run with and without heating. Accumulation of sintering record and confirmation of sintering atmosphere for the desired oxygen partial pressure will lead to the next stage task, fabrication of MOX fuel with target stoichiometry. Surrogate samples from depleted $\mathrm{UO}_{2}$ will be used for the reproducibility confirmation experiments and basic MOX composition, $\left(\mathrm{U}_{0.8} \mathrm{Pu}_{0.2}\right) \mathrm{O}_{2}$, will be used for the sintering conditioned which requires verification of sintered sample properties.

Other sintering parameters to be explored will include the effect of heating rate and employment of a binder burn out step. So far, the heating and cooling rate used for the ATP runs was as high $15^{\circ} \mathrm{C} / \mathrm{m}$. This rate was used to ensure the furnace is capable of high heating and cooling rates as well as to incorporate the processing time within the FMF operating hours. However, the normal heating rate of MOX for the stoichiometry control is $2 \sim 5^{\circ} \mathrm{C} / \mathrm{m}$ range. The heating rate needs to be slow enough to provide enough time for the reaction to meet the target stoichiometry along with relaxing the strain resulting from the substoichiometry. The need of treatment of condensed volatiles during the binder burn out process will be considered as well.

\subsection{Oxygen Partial Pressure and Stoichiometry Relationship Map Development}

The stoichiometry of the sintered MOX pellet is driven by the oxygen partial pressure during the sintering. The relationship between oxygen partial pressure and the resulting stoichiometry of the sintered pellet needs to be verified for the specific fabrication equipment. The factors to be considered in this investigation are fuel composition, sintering temperature, oxygen partial pressure and stoichiometry of the sintered pellets. The final goal of this task is to establish a specific sintering atmosphere for a given composition and sintering temperature. Characterization of sintered MOX pellet stoichiometry will be an important issue for establishing the fabrication condition vs stoichiometry relationship, and will be treated as a separate task. In order to characterize the stoichiometry of sintered MOX, the sample needs to be heated to $\sim 900^{\circ} \mathrm{C}$ in an oxygen potential for 2.0 stoichiometry. The oxygen potential for the 2.0 stoichiometry is reported to be $-420 \mathrm{~kJ} / \mathrm{mol}$ for $\mathrm{UO}_{2}$ case, but, for MOX, it is influenced by the existence of minor actinides. It is reported that the oxygen partial pressure for 2.0 stoichiometry is $\sim-350 \mathrm{~kJ} / \mathrm{mol}$ of $\left(\mathrm{U}_{0.8} \mathrm{Pu}_{0.2}\right) \mathrm{O}_{2}$ composition for the temperature range of $1200 \sim 1350^{\circ} \mathrm{C}$. The weight change resulted from such a heat treatment converting the sample to 2.0 stoichiometry will represent the stoichiometry of the sample before heat treatment. Further details of the stoichiometry characterization will be discussed in Section 2-5.

$\left(\mathrm{U}_{0.8} \mathrm{Pu}_{0.2}\right) \mathrm{O}_{2}$ composition will be used for the initial process map generation. The process map will then be further expanded to include the compositions with 2 3\% of Am and Np replacing U. Task 2-2 using surrogate composition will effectively reduce the number of sintering runs required for this task.

\subsection{High Temperature Stable Microstructure Development}

The target relative density of sintered MOX is $85 \sim 90 \%$ of the theoretical density. The high operating centerline temperature of the fast reactor, in excess of $2000^{\circ} \mathrm{C}$, can cause the densification and, consequently, dimensional changes of the fuel. In order to prevent the densification of the fuel during the operation, a stable microstructure is required. A low density, stable at higher temperatures than sintering temperature $\left(\sim 1600^{\circ} \mathrm{C}\right)$ microstructure can be achieved by employing a 'constrained' sintering concept. A green powder mixture containing dense inclusions results in a low sintered density due to the formation of a ridged network leaving porous area inside the network. 
Inclusions will be prepared by crushing sintered pellets of the identical composition sintered to 95\% relative density. Through grinding and sieving, 20 40 $\mu \mathrm{m}$ inclusions will be prepared and mixed with raw powders. The weight fraction of the inclusions will be in the range of $10-25 \%$ and powder mixing will be conducted in the same manner as the original process. Sintered density and physical appearance will be the main characteristics of this task. Monitoring dimension and density changes of the sintered samples after post-sintering heating to $2000^{\circ} \mathrm{C}$ could be conducted after fabrication of successful samples. Both surrogate and basic MOX compositions will be used for this task, and feasibility will be confirmed by making surrogate samples in 2018. Density measurement will basically depend on geometric method. Using Archimedes method will be considered, if needed after locating proper liquids which can be handled inside the glove box.

\subsection{Stoichiometry Characterization Method Development}

The current characterization method using a TGA instrument uses dry Ar mixed with 6\% hydrogen. Previous data from depleted uranium oxide samples with known stoichiometries indicated that samples converted to a 2.0 stoichiometry after $24 \mathrm{hrs}$ at $850^{\circ} \mathrm{C}$. The result matched well with expectation that $\mathrm{UO}_{2}$ will not reduce to below a 2.0 stoichiometry. However, in case of MOX with the existence of minor actinide, stoichiometry can go below 2.0 depending on the atmosphere. Therefore, a controlled atmosphere, similar to what was developed for the sintering furnace, is required for the stoichiometry characterization of the MOX samples. Samples will be heated under the atmosphere with oxygen partial pressure equivalent to 2.0 stoichiometry as presented in the Table 2 . The gas mix ratio for $-350 \mathrm{~kJ} / \mathrm{mol}$ which is equivalent to 2.0 stoichiometry varies with temperature as shown in Table 2 . The stoichiometry can be determined from the following equation:

$$
\mathrm{O} / \mathrm{M}=2.000-\operatorname{At}(\mathrm{MOX}) / \operatorname{At}(O) \times \Delta w / w
$$

where, $\operatorname{At}(\mathrm{MOX})$ is atomic weight of $\mathrm{MOX}$ with 2.0 stoichiometry, $\operatorname{At}(\mathrm{O})$ is atomic weight of oxygen, $\Delta \mathrm{w}$ is the weight change from the heat treatment and $\mathrm{w}$ is the weight of the sample after the heat treatment. (modified from the ISO 21484, Nuclear Fuel Technology- Determination of the O/M ratio in MOX pellets- Gravimetric method and “Oxygen Potential of Mixed Oxide Fuels for Fast Reactors", Journal of Nuclear Materials 2009, pp419-423, M. Kato et al.)

Table 2. Gas mixture ratio and for $-350 \mathrm{~kJ} / \mathrm{mol}$ oxygen partial pressure for $\left(\mathrm{U}_{0.8} \mathrm{Pu}_{0.2}\right) \mathrm{O}_{2}$ composition at various temperatures.

\begin{tabular}{|c|c|c|}
\hline $\mathrm{T}\left({ }^{\circ} \mathrm{C}\right)$ & $\mathrm{PO}_{2}$ & $\mathrm{H}_{2} / \mathrm{H}_{2} \mathrm{O}$ ratio \\
\hline 700 & $1.636 \mathrm{E}-19$ & 0.1095 \\
\hline 800 & $9.214 \mathrm{E}-18$ & 0.2577 \\
\hline 900 & $2.610 \mathrm{E}-16$ & 0.5230 \\
\hline
\end{tabular}

Two parallel approaches will be used for establishing the stoichiometric characterization; acquiring the mass flow system and oxygen sensing capability to the TGA in the Analytical Lab (depending on programmatic direction and funding) and utilizing tube furnace in FMF glove box. The oxygen sensing capability to the tube furnace is already provided. However, providing condition for measuring the sample weight to second decimal of $\mathrm{mg}$ range $(.00001 \mathrm{~g})$ will be one of the main issues in utilizing the tube furnace. Reliability and reproducibility of the current method will be verified using samples of surrogate and basic MOX composition. The effect of Pu on the oxygen partial pressure required for the control stoichiometry will be compared to surrogate case.

\section{CONCLUSION}

The atmosphere control is an essential part of fabrication of MOX fuels with target stoichiometry. Newly added capabilities for controlling and monitoring oxygen partial pressure during the sintering of MOX 
pellet have been tested and confirmed for the basic functions. The pellets fabricated through the ATP resulted in low green and sintered relative densities, 45 and 74\%, respectively. The objective of this plan is to further develop a robust fabrication process of MOX fuel with desired properties. The tasks include efforts for improving density, establishing a stable oxygen partial pressure control, understanding and correlating processing condition and properties, obtaining a high temperature stable microstructure, and development of stoichiometry characterization procedures. 